\title{
A Randomized, Double-Blind, Placebo-Controlled Clinical Trial of Tocilizumab, An Interleukin-6 Receptor Antibody, For Residual Symptoms in Schizophrenia
}

\author{
Ragy R Girgis*, 1,2, Adam Ciarleglio ${ }^{1,2}$, Tse Choo ${ }^{1,2}$, Gregory Haynes ${ }^{1,2}$, Joan M Bathon ${ }^{3}$, Serge Cremers ${ }^{4}$, \\ Joshua T Kantrowitz ${ }^{1,2,5}$, Jeffrey A Lieberman ${ }^{1,2}$ and Alan S Brown ${ }^{1,2}$ \\ 'Department of Psychiatry, College of Physicians and Surgeons, Columbia University, New York, NY, USA; ${ }^{2}$ New York State Psychiatric Institute, \\ New York, NY, USA; ${ }^{3}$ Department of Medicine, Columbia University, New York, NY, USA; ${ }^{4}$ Department of Pathology, Columbia University, New \\ York, NY, USA; ${ }^{5}$ Schizophrenia Research Center, Nathan Kline Institute for Psychiatric Research, Orangeburg, NY, USA
}

\begin{abstract}
Evidence from preclinical, epidemiological, and human studies indicates that inflammation, and in particular elevated interleukin-6 (IL-6) activity, may be related to clinical manifestations and pathophysiology of schizophrenia. Furthermore, studies in preclinical models suggest that decreasing IL-6 activity may mitigate or reverse some of these deficits. The purpose of this trial was to test whether an IL-6 receptor antibody, tocilizumab, would improve residual positive and negative symptoms and cognitive deficits in schizophrenia. We randomized 36 clinically stable, moderately symptomatic (i.e., Positive and Negative Syndrome Scale (PANSS) > 60) individuals with schizophrenia to 3 monthly infusions of $8 \mathrm{mg} / \mathrm{kg}$ tocilizumab or placebo (normal saline). The primary outcome was effect at week 12 on the PANSS Total Score. Effects on the MATRICS, other PANSS subscales, Clinical Global Impression, and Global Assessment of Functioning were secondary outcomes. There were no observed treatment effects on any behavioral outcome measure. Baseline C-reactive protein (CRP) or cytokine levels did not predict treatment outcome, nor were there correlations between changes in these inflammatory markers and the measured outcomes. As expected, IL-6 and IL-8 increased, while CRP decreased, in the tocilizumab group compared with the placebo group. This study did not reveal any evidence that an IL-6 receptor antibody affects behavioral outcomes in schizophrenia. One potential explanation is the lack of capacity of this agent to penetrate the central nervous system. Additional trials of medications aimed at targeting cytokine overactivity that act directly on brain function and/or treatment in early-stage psychosis populations are needed. Neuropsychopharmacology (2018) 43, I317-1323; doi:I0. I038/npp.2017.258; published online 13 December 2017
\end{abstract}

\section{INTRODUCTION}

Links between in utero early life, infection, and inflammation and the later development of schizophrenia (SZ) have been postulated for years. Initial studies using ecologic data on epidemics of infection reported associations between second trimester influenza exposure with SZ (Adams et al, 1993; Brown and Derkits, 2010; O'Callaghan et al, 1991). Subsequent birth cohort studies further identified associations between $\mathrm{SZ}$ and early pregnancy exposure to infectious agents, including influenza (Brown et al, 2004), herpes simplex virus type 2 (Buka et al, 2001, 2008), and Toxoplasma gondii (Brown et al, 2005; Mortensen et al, 2007). Several models have attempted to explain how prenatal or lifetime infection increase the risk of SZ (Brown and Derkits, 2010). The most parsimonious of these

*Correspondence: Dr RR Girgis, Department of Psychiatry, Columbia University College of Physicians and Surgeons, 105I Riverside Drive, Unit 31, New York 10032, NY, USA. Tel: +I 646774 5553; Fax: + | 646774 5237; E-mail: ragy.girgis@nyspi.columbia.edu Received 30 July 2017; revised I October 2017; accepted 24 October 2017; accepted article preview online I November 2017 suggests that there are common effects of infection that may increase the risk of SZ (Brown and Derkits, 2010). The induction of proinflammatory cytokines, a family of soluble proteins, are considered to be one of the most likely candidate mediators (Gilmore and Jarskog, 1997) that have an important role as the systemic mediators of host response to infection (Brown and Derkits, 2010). These molecules, which include interleukin-6 (IL-6), can be produced in the CNS, have numerous effects on both glial and neuronal components of the CNS and alter both normal and abnormal brain development (Benveniste et al, 1990; Frei et al, 1989; Gilmore and Jarskog, 1997; Hopkins and Rothwell, 1995; Rothwell and Hopkins, 1995). IL-6 in particular is implicated by preclinical studies that show that offspring of rodents exposed to prenatal infections, IL-6, or agents that evoke robust, antipathogen immune responses (e.g., lipopolysaccharide) develop behavioral (e.g., hyperlocomotor), electrophysiological (e.g., deficits in prepulse inhibition), and biochemical (e.g., elevated IL-6 levels) effects characteristic of SZ, which are either mitigated by treatment with anti-IL-6 agents or prevented in IL-6 knockout animals (Ashdown et al, 2006; Borrell et al, 2002; Fortier et al, 2004; Patterson, 
2009; Samuelsson et al, 2006; Smith et al, 2007). Therefore, cytokines, and IL-6 in particular, may mediate the association between infection and SZ.

There is also substantial evidence from studies in patients that supports a connection between alterations in IL-6 levels, as well as abnormalities in inflammation, to SZ. Miller et al (2011) reported, in a meta-analysis, that IL-6 levels were elevated in the plasma of both first-episode (effect size $=1.4$ ) and acute relapsed (effect size $=0.96$ ) patients, whereas IL-6 levels significantly decreased after treatment (effect size $=$ - 0.31) (Miller et al, 2011). These data suggest that IL-6 is a state marker of SZ, normalizing with treatment. Evidence from studies in which antipsychotic medications were added to blood from healthy control subjects that had been stimulated by toxic shock syndrome toxin, and therefore had elevated IL-6 levels, had no effect on plasma IL-6 levels (Himmerich et al, 2011), further suggesting that the effects of antipsychotic treatment on IL-6 levels in SZ reported by Miller et al (2011) are specific to SZ, and may be related to an ongoing, underlying persistent inflammatory process that can be ameliorated by treatment.

Treatment studies of anti-inflammatory agents such as celecoxib (Akhondzadeh et al, 2007; Muller et al, 2002, 2010) and aspirin (Laan et al, 2010) as add-on treatment to antipsychotic agents revealing improvements on the Positive and Negative Syndrome Scale (PANSS) and Clinical Global Impression (CGI) scales also support the development of treatments aimed at reducing inflammation.

In spite of these promising findings, it is notable that symptom improvement in these studies was modest (Nitta et al, 2013). Moreover, these anti-inflammatory medications target cyclooxygenases, leading to reductions in prostaglandins. In contrast to cytokines, there is no direct evidence of associations between cyclooxygenases and prostaglandins in SZ. Consequently, pharmacologic agents that potently inhibit the activity of specific cytokines, in particular IL-6, represent a novel approach to the treatment of SZ and may offer greater therapeutic benefit. Advances in the treatment of rheumatologic diseases have made the investigation of such treatment now possible.

Tocilizumab (TCZ; Actemra $\odot$ ) is FDA approved for rheumatoid arthritis in individuals who have not responded to at least one TNF- $\alpha$ therapy, and for juvenile idiopathic arthritis. TCZ is a humanized monoclonal antibody against the IL-6 receptor and is administered as a once monthly intravenous injection. Its benefit for rheumatoid arthritis symptoms is dose dependent and may occur within 1 week of treatment (Burmester et al, 2011; Smolen et al, 2007), although the effects of immunologic agents on cytokine profiles in rheumatoid arthritis are quite variable (Amital et al, 2007; Wright et al, 2012). Importantly, the IL-6 receptor is also particularly suited as a target for treatment development in SZ and in general due to the existence of a soluble IL-6 receptor (i.e., that uses 'trans' signaling) in addition to a typical membrane-bound receptor (i.e., that utilizes ' $c i s$ ' signaling). This lends several advantages to the IL-6 system, including protecting IL- 6 and activating cells that would not otherwise respond to IL-6 (as reviewed in Jones et al, 2011). Recent evidence supports this approach of using a cytokine antagonist for psychiatric illness. Infliximab, a TNF- $\alpha$ antibody, was administered intravenously at baseline, 2 weeks, and 6 weeks to individuals with treatment resistant depression. While infliximab did not show overall improvement on depressive symptomatology compared with placebo, there was an association between increasing baseline C-reactive protein and response to infliximab in treatmentresistant depression (Raison et al, 2012). Although infliximab is not known to cross the blood-brain barrier (BBB), this study indicates that direct action on the CNS is not necessary for a behavioral response to the medication. Therefore, in this trial, we examine whether an IL-6 receptor antibody, TCZ, would improve residual positive and negative symptoms and cognitive deficits in SZ.

\section{MATERIALS AND METHODS}

All procedures were approved by the Institutional Review Board of the New York State Psychiatric Institute (NYSPI) at the Columbia University Medical Center between March 2014 and January 2017. All subjects provided written, informed consent for inclusion in this study. Subjects were 36 medically healthy, adult (age 18-59 years) patients with SZ or schizoaffective disorder who were stably treated for at least 1 month without changing their dose of antipsychotic medication. They had a baseline PANSS of $>60$. Concomitant medications were permitted as long as patients were on stable doses for at least 1 month. Subjects could not have been taking clozapine or any medication that has a potential for neutropenia or thrombocytopenia, nor could they have had any chronic inflammatory disorder or have had any recent or severe infectious condition. Individuals with autoimmune diseases were excluded, as well as individuals with any substance use (confirmed by urine toxicology at each visit). One subject in each group was taking a baby aspirin. No other anti-inflammatory medications were taken by any subject in this trial. One subject in the TCZ group changed their dose of antipsychotic from 2 to $5 \mathrm{mg}$ of Abilify. There were no other changes in psychotropic medications for the duration of the trial.

\section{Randomization, Masking, and Assessment Procedures}

Baseline assessments (see 'Assessments' below) were completed within 2 weeks of the first study drug administration. After the baseline procedures, patients were randomized 1:1 in a double-blind design to placebo or $8 \mathrm{mg} / \mathrm{kg}$ TCZ ( $\max$ dose $800 \mathrm{mg}$ ). This dose was chosen to maximize target engagement. While the recommended starting dose of TCZ is $4 \mathrm{mg} / \mathrm{kg}$, we chose to use $8 \mathrm{mg} / \mathrm{kg}$ as a starting dose to maximize target engagement since all subjects were receiving a maximum of three doses. Randomization was not stratified. The dose of TCZ or placebo was decreased as necessary for tolerability.

After randomization, each subject received three monthly doses of TCZ or placebo. At weeks 2, 4, 8, and 12, all subjects completed the assessment battery (see below) and had safety assessments (i.e., blood, urine, EKG, EPS rating scales).

\section{TCZ/Placebo Administration}

Study drug was administered in $100 \mathrm{~cm}^{3}$ normal saline as a constant intravenous drip over $60 \mathrm{~min}$. The subjects received constant monitoring by an ACLS-certified physician during the infusion and for $60 \mathrm{~min}$ afterwards, to monitor for 


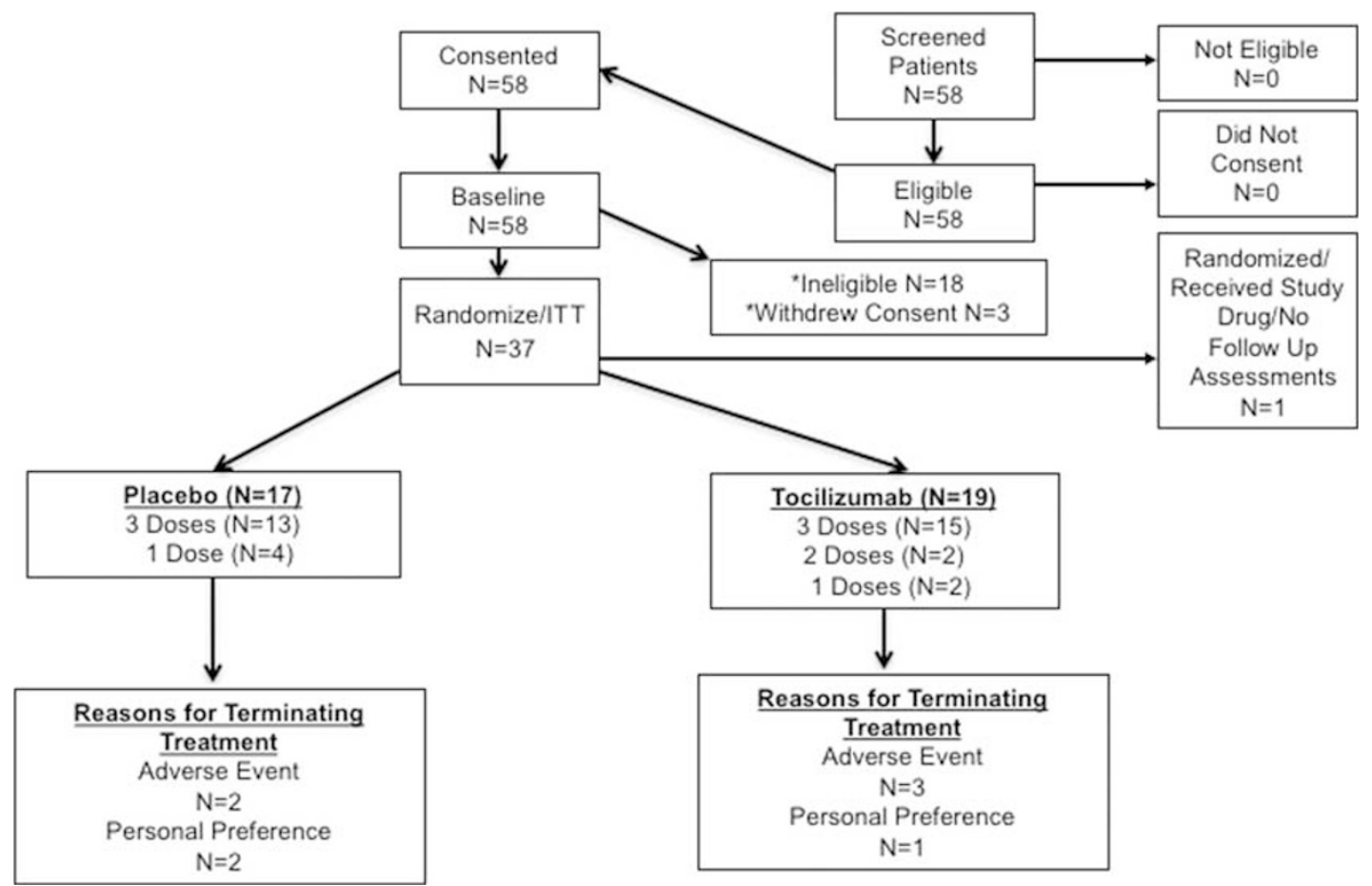

Figure I Consort patient flow diagram.

allergic reactions. Blood pressure and heart rate were obtained before and right after the infusion. Before each infusion and at week 12, blood samples were obtained for CRP (C-reactive protein), GM-CSF (granulocyte-macrophage colony-stimulating factor), IFN- $\gamma$ (interferon- $\gamma$ ), TNF- $\alpha$ (tumor necrosis factor- $\alpha$ ), IL-1b, IL-2, IL-6, IL-8, IL-10, IL-12, and IL-17a.

\section{Assessments}

The primary symptom assessment was the PANSS, which assesses severity of positive, negative, and cognitive symptoms in SZ (Kay and Sevy, 1990), with secondary measures of the Global Assessment of Functioning (GAF) scale (Revheim and Medalia, 2004), which is a clinician-rated measure of symptom severity and role function, and the CGI-Severity and -Improvement Scales (CGI-S and CGI-I) (Guy, 1976), which are clinician-rated measures of overall clinical status. Cognition was assessed using the MATRICS consensus cognitive battery (Green et al, 2004; Marder and Fenton, 2004). Safety was assessed with laboratory evaluation, physical examination, EKG, the SAFTEE (Levine and Schooler, 1986) to assess general side effects, the Simpson Angus Scale (SAS) to assess extrapyramidal symptoms (Simpson and Angus, 1970), and the Columbia Suicide Severity Rating Scale (C-SSRS) (Posner et al, 2011) for suicide risk. The clinical trial was monitored by an independent DSMB.

\section{Data Analysis}

The primary outcome measure was total PANSS score. Secondary outcome measures correspond to the other assessments described above.
Linear mixed-effects models were used to assess the effect of treatment on each outcome measure. Each outcome was modeled as a function of the baseline value of the outcome, visit time, and treatment group. Each model included a random intercept (Fitzmaurice et al, 2004) to account for the within-subject correlation among repeated measures. To investigate potential moderating effects of the baseline cytokine measures, similar mixed-effects models were fit that additionally included baseline cytokine value and baseline cytokine value $\times$ treatment group interaction terms. Baseline cytokine measures were log-transformed before entering the models. PROC GLIMMIX in SAS was used to conduct these analyses.

Cytokine value (log-transformed) change scores were calculated at each assessment timepoint during the study by subtracting the baseline measure from each subsequent measure. The change scores were compared between the treatment groups at each post-baseline time point via twosample $t$-tests.

All analyses were conducted based on the intent-to-treat (ITT) principle. All statistical tests were two-tailed and employed an $\alpha$ significance level of 0.05 .

This trial was registered at clinical trials.gov (NCT02034474; https://clinicaltrials.gov/ct2/show/NCT02034474).

\section{RESULTS}

As shown in Figure 1, of the 58 subjects enrolled in this trial, 37 were randomized, one was excluded due to use of marijuana during the trial, and thus 36 were included in the ITT analysis. Psychotropic medications taken by the control subjects included: haloperidol (2), aripiprazole (4), olanzapine (2), perphenazine (1), paliperidone (1), fluphenazine (1), quetiapine (3), and risperidone (4). Psychotropic medications taken by the TCZ subjects included: chlorpromazine (1), 
paroxetine (1), bupropion (1), benztropine (1), lurasidone (3), risperidone (4), olanzapine (3), aripiprazole (4), haloperidol (2), ziprasidone (1), trazodone (1), lithium (1), sertraline (1), paliperidone (1), and quetiapine (2). The demographics of the complete ITT sample are provided in Table 1. Treatment groups were comparable with respect to demographic factors, behavioral measures, and cytokine values.

There were no significant treatment effects for any of the behavioral outcomes (PANSS including positive and general subscales, MATRICS including subscales, CGI, GAF), with the exception of a significant treatment effect on the PANSSnegative subscale such that those on TCZ scored 1.93 points higher on average over time than those in the placebo group $(p=0.044)$. Additional analyses investigating PANSS total change score showed a significant reduction in the placebo group at week 2, with an average decrease of 9.88 $(\mathrm{SD}=11.74)$, while there was little to no change in the TCZ group ( $p$-value for difference in the groups' change scores at week $2=0.007$ ). For descriptive purposes, we include Supplementary Table 1 that gives the raw mean (SD) values for each behavioral outcome measure at each visit as well as raw mean (SD) change score from baseline at each post-baseline visit. P-values, based on Wilcoxon's signedrank tests, for testing within-group changes from baseline at each post-baseline visit are provided for each group. $P$ values, based on Wilcoxon's rank-sum tests, for testing between group differences in mean change scores are also provided.

The models that included the interaction between baseline cytokine measures and treatment revealed several cytokine measures that may be potential moderators of treatment effect with respect to the primary and secondary outcomes. Table 2 shows each outcome with its corresponding treatment effect moderator for those models in which the interaction between baseline cytokine measures and treatment was significant at the 0.05 level. In Table 2, Placebo Slope gives the effect of the baseline cytokine value on the outcome for those treated with placebo and TCZ Slope gives the same for those treated with TCZ. The table shows, for example, that for those on placebo, a one-unit increase in baseline GM-CSF (on log-scale) corresponds to a decrease of 1.54 in PANSS total on average over time, whereas for those on TCZ, a one-unit increase in baseline GM-CSF (on logscale) corresponds to a decrease of 8.79 in PANSS Total on average over time. Other rows of the table can be interpreted similarly. We note that none of these interactions are significant after correcting for multiple testing based on the number of models fit and tests conducted. We present the results for descriptive purposes.

There was no evidence of differences in relationships (as measured by Pearson's correlation coefficients) between change in CRP (log-transformed) from baseline to 12-week follow-up and change in any of the behavioral measures from baseline to 12 -week follow-up between treatment groups.

Finally, as expected, IL-6 and IL-8 levels significantly increased in the TCZ group over time, and CRP levels decreased over time, while these measures remained unchanged from baseline over time in the placebo group (Table 3). There were no differences in the changes in levels of the other cytokines over time between the treatment groups.
Table I Baseline Characteristics for the Complete ITT Sample

\begin{tabular}{lcc}
\hline & $\begin{array}{c}\text { Placebo (N= I7), } \\
\text { mean (SD) or \% }\end{array}$ & $\begin{array}{c}\text { Tocilizumab (N= I9), } \\
\text { mean (SD) or \% }\end{array}$ \\
\hline Age (years) & $41.67(10.25)$ & $43.24(11.42)$ \\
Gender (male) & $76.47 \%$ & $63.16 \%$ \\
PANSS Total & $73.94(15.52)$ & $70.68(11.20)$ \\
Smoker (yes) & $41.18 \%$ & $42.11 \%$ \\
Cigarettes/day & $8(3)$ & $10(5)$ \\
Chlorpromazine & $377(346)(\mathrm{N}=12)$ & $257(192)(\mathrm{N}=15)$ \\
Equivalents (mg) & & \\
Race & & $15.79 \%$ \\
Cauc. & $52.94 \%$ & $57.89 \%$ \\
Afr. American & $35.29 \%$ & $0.00 \%$ \\
Asian & $5.88 \%$ & $5.26 \%$ \\
Hispanic/Afr. American & $0.00 \%$ & $10.53 \%$ \\
Hispanic/Cauc. & $0.00 \%$ & $10.53 \%$ \\
Hispanic/Mixed & $5.88 \%$ & $218.63(45.24)$ \\
Weight (pounds) & $203.24(51.95)$ & $4.00(0.33)$ \\
CGl-Severity & $4.18(0.39)$ & \\
\hline
\end{tabular}

Table 2 Baseline Cytokine Moderators of Treatment Effect

\begin{tabular}{lcrrr}
\hline Outcome & $\begin{array}{c}\text { Moderator } \\
\text { (log- } \\
\text { transformed) }\end{array}$ & $\begin{array}{c}\text { Placebo } \\
\text { Slope (SE) }\end{array}$ & $\begin{array}{c}\text { Tocilizumab } \\
\text { Slope (SE) }\end{array}$ & p-value \\
\hline PANSS Total & GM_CSF & $-1.54(\mathrm{I} .66)$ & $-8.79(3.09)$ & 0.0385 \\
PANSS Positive & GM_CSF & $-0.78(0.53)$ & $-3.57(0.99)$ & 0.0121 \\
CGI-Severity & IL_I0 & $0.20(0.09)$ & $-0.01(0.05)$ & 0.0425 \\
CGI-Severity & IL_I7A & $0.15(0.06)$ & $-0.02(0.04)$ & 0.0217 \\
CGI-Severity & IL_8 & $0.20(0.06)$ & $0.03(0.05)$ & 0.039 \\
CGI-Severity & TNFa & $0.49(0.14)$ & $0.01(0.07)$ & 0.0031 \\
BAC_SC_t_score & IL_Ib & $-9.49(4.56)$ & $1.39(2.56)$ & 0.0412 \\
\hline
\end{tabular}

Placebo Slope (SE) and Tocilizumab Slope (SE) give the estimated effects of the specified baseline cytokine on the specified outcome in the placebo and tocilizumab groups, respectively. $P$-value is for the baseline cytokine $\times$ treatment interaction term in the moderator model.

\section{Tolerability and Adverse Effects}

TCZ and placebo were well tolerated and all adverse events were mild. In the placebo group, one subject developed an upper respiratory tract infection and a postinfectious asthma that was treated with prednisone, and one patient developed a tooth infection requiring treatment with antibiotics. One subject in each group was hospitalized for worsening schizophrenia. In the TCZ group, one patient developed nausea and one fatigue. All adverse events fully resolved within days. Regarding effects on neutrophil counts, two individuals in the placebo group had absolute neutrophil counts (ANC) that dropped into the 1000-2000 cells/ $\mu$ l range (one decreased his placebo dose from 8 to $4 \mathrm{mg} / \mathrm{kg}$ and the other withdrew). Two individuals in the TCZ group had ANCs that dropped into the 1000-2000 cells/ $\mu$ l range (both decreased their study drug dose from 8 to $4 \mathrm{mg} / \mathrm{kg}$ ). Another subject in the TCZ group developed agranulocytosis (ANC of 
Table 3 Change in CRP, IL-6, and IL-8 Levels (log-transformed) Over Time

\begin{tabular}{lcccc}
\hline Cytokine & Week & $\begin{array}{c}\text { Placebo, mean } \\
\text { (SD) }\end{array}$ & $\begin{array}{c}\text { Tocilizumab, mean } \\
\text { (SD) }\end{array}$ & p-value \\
\hline CRP & 0 & $0.7 \mid(1.26)$ & $1.11(1.46)$ & \\
& 4 & $0.97(1.74)$ & $-0.47(1.67)^{\mathrm{a}}$ & 0.0046 \\
& 8 & $0.85(1.21)$ & $-0.53(1.42)^{\mathrm{a}}$ & 0.0003 \\
& 12 & $0.90(1.46)$ & $-0.35(1.60)^{\mathrm{a}}$ & 0.0090 \\
& & & & \\
IL-6 & 0 & $1.28(0.92)$ & $1.56(1.38)$ & \\
& 4 & $1.49(1.06)$ & $2.96(0.86)^{\mathrm{a}}$ & $<0.0001$ \\
& 8 & $1.32(1.12)$ & $2.63(1.22)^{\mathrm{a}}$ & $<0.0001$ \\
& 12 & $1.74(0.59)$ & $2.73(1.06)^{\mathrm{a}}$ & 0.0082 \\
& & & & \\
IL-8 & 0 & $1.53(0.70)$ & $1.45(0.76)$ & \\
& 4 & $1.51(0.76)$ & $1.73(0.73)^{\mathrm{a}}$ & 0.0088 \\
& 8 & $1.49(0.88)$ & $1.65(0.73)^{\mathrm{a}}$ & 0.0806 \\
& 12 & $1.48(0.91)$ & $1.80(0.79)^{\mathrm{a}}$ & 0.0147 \\
\hline
\end{tabular}

$P$-values are for testing if change from baseline to each visit is different between the treatment groups.

andicates a p-value $<0.05$ for testing the within-group change.

400 cells $/ \mu \mathrm{l}$ ) after two doses of study drug and was withdrawn from the trial. No subjects had any clinical sequelae from their decreases in ANC and all ANC returned to normal without additional intervention. One subject in the TCZ group was discontinued due to elevated liver function tests and another had elevated liver function tests, remained in the trial, and decreased their dose of study drug from 8 to $4 \mathrm{mg} / \mathrm{kg}$. No subjects had any clinical sequelae from their elevations in liver function tests and all liver function tests returned to normal without intervention.

\section{DISCUSSION}

This clinical trial of the effects of an IL-6 receptor antibody on positive and negative symptoms and cognitive deficits in SZ was negative. There were no main effects of treatment nor were there drug-related effects correlated with CRP or cytokine levels at baseline or during treatment. While other trials examining the effects of anti-inflammatory agents such as celecoxib (Akhondzadeh et al, 2007; Muller et al, 2002, 2010) and aspirin (Laan et al, 2010) have been performed, this was the first controlled, clinical trial of an agent that specifically targets a cytokine in SZ, and is consistent with a recent open-label trial of $4 \mathrm{mg} / \mathrm{kg}$ TCZ in five subjects with SZ that observed no significant improvement in psychopathology scores (Miller et al, 2016). These results are also consistent with recent PET studies of neuroinflammation showing equivocal results in studies comparing high risk, first episode, and chronic patients with control subjects (Bloomfield et al, 2016; Collste et al, 2017; Hafizi et al, 2017a, b; Hafizi et al; Kenk et al, 2015).

The rationale for using an IL-6 receptor antibody in SZ is well supported by previous work. Behrens et al (2008) hypothesized a connection between IL-6 and the psychotomimetic effects of ketamine. They found that, in mice, ketamine disrupts parvalbumin containing interneurons $(\mathrm{PV}+)$, aberrations of which have been implicated in SZ (Lewis et al, 2005) through activation of NADPH oxidase (Behrens et al, 2007), an effect that was mimicked by IL-6 administration and reversed by neutralization of NADPH oxidase (Behrens et al, 2008). Ketamine administration also induces expression of IL-6, and IL-6 antibodies abolish the effects of ketamine on PV+ interneurons (Behrens et al, 2008). The authors also reported that, in IL-6 knockout mice, ketamine did not alter PV+ interneurons nor did it activate NADPH oxidase. These findings suggest that IL- 6 may be a mediator of the deleterious effects of ketamine on PV+ interneurons, which are critical in the regulation of pyramidal cell activity and possibly more directly in cognitive processes such as working memory (Lewis et al, 2005). Knocking out or neutralizing IL-6 would be expected to mitigated these abnormalities, further supporting IL-6 as a prime target for drug development in SZ.

One potential reason for the negative results is that TCZ does not cross the BBB. However, there are two main mechanisms whereby TCZ may affect CNS function. First, in the CNS, the effects of IL-6, whether of central or, less commonly, peripheral (Reyes and Coe, 1998; Schwarz, 2003), origin, are primarily mediated by soluble IL-6 receptors, rather than membrane-bound receptors. Soluble IL-6 receptors readily cross the $\mathrm{BBB}$ and, when peripherally injected into mice, localize in cortico-striatal-thalamocortical circuits in the brain (Patel et al, 2012), areas of prime importance in SZ. TCZ would bind these receptors and prevent them from traversing the $\mathrm{BBB}$ and exerting their effects on the CNS. Second, cytokines such as IL-6 may affect the CNS via activating a proinflammatory cascade both peripherally and centrally (Reyes and Coe, 1998). TCZ, by binding the IL- 6 receptor, would prevent this cascade and the release of other cytokines that would themselves have the ability to act on the CNS. These two mechanisms of action of TCZ would effectively neutralize IL-6 regardless of its origin (i.e., central or peripheral). Therefore, despite the inability of TCZ to penetrate the $\mathrm{BBB}$, it would be predicted to effectively neutralize the effects of IL-6 in the CNS. In this trial, TCZ did clearly engage IL- 6 receptors as demonstrated by its effects on IL-6 and CRP. However, whether or not its ability to engage the IL-6 receptor peripherally, and not centrally, is adequate to produce an effect in SZ remains unknown.

It may also be the case that effects of TCZ would have been observed had we examined an unmedicated, acute, and/or first episode population. This is supported by Miller et al (2011). who reported, in a meta-analysis, that IL-6 levels were elevated in the plasma of both first-episode (effect size $=1.4$ ) and acute relapsed (effect size $=0.96$ ) patients, while IL-6 levels significantly decreased after treatment (effect size $=-0.31$ ). These data suggest that IL-6 is a state marker of SZ, normalizing with treatment. Alternatively, elevated inflammatory markers in chronic SZ may not necessarily be causal. Conceivably, elevated IL-6 may have had earlier detrimental neurodevelopmental effects that are resistant to treatment, necessitating preventive therapy before illness onset, such as during the premorbid or prodromal periods. Elevated IL-6 originating during the prenatal period might reflect an infectious or inflammatory process in the mother. It is also possible that our sample size 
was too small to detect an effect. In particular, it is possible that enriching for elevated baseline CRP, as suggested by Raison et al (2012), would have increased our chances of observing an effect. Additionally, the placebo response in this trial is similar to what has been observed in recent years and may have limited our ability to observe TCZ treatment effects (Kemp et al, 2010; Kinon et al, 2011; Rutherford et al, 2014). Further, although in RA early improvements tended to persist (Burmester et al, 2011), it is possible that improvements occurred within the first 2 weeks after the infusion (Miller et al, 2016), although these also tended to persist. In addition, while there were no significant differences in race/ethnicity between groups, there are known biological and psychosocial differences in the epidemiology of inflammatory diseases that are important to consider (Ghodke et al, 2005; McBurney and Vina, 2012). Finally, it is possible that a PANSS cutoff of 60 represented too mild a level of symptoms to observe an effect.

In summary, we observed no effects of an IL-6 receptor antibody on positive or negative symptoms or cognitive deficits in chronic SZ. We suggest future trials of these and other biologics on prodromal or acutely ill cases populations, as well as agents with the capacity to cross the BBB.

\section{FUNDING AND DISCLOSURE}

This trial was supported by a grant from the Stanley Medical Research Institute (no. 12T-004). TCZ for this trial was donated by Genentech. Dr Girgis has also received research support from Allergan, Otsuka, and BioAdvantex. Dr Bathon consults to Regeneron and receives <US\$5000/year for this. Dr Kantrowitz reports having received consulting payments within the past 24 months from Krog \& Partners Incorporated, Kinetix Group, Annenberg Center for Health Sciences at Eisenhower, Semantics MR, Transperfect, and Cowen and Company. He has conducted clinical research supported by the NIMH, the Stanley Foundation, Taisho, Lundbeck, Merck, and Lilly within the past 24 months. He owns a small number of shares of common stock in GlaxoSmithKline. Dr Lieberman has received support administered through his institution in the form of funding or medication supplies for investigator-initiated research from Denovo, Taisho, Pfizer, Sunovion, and Genentech, and for company sponsored phase II, III and IV studies from Alkermes and Allergan, and is a is a consultant to or member of the advisory board of Intracellular Therapies, Lilly, Pierre Fabre, and Psychogenics. He neither accepts nor receives any personal financial remuneration for consulting, speaking, or research activities from any pharmaceutical, biotechnology, or medical device companies. He has received honoraria for serving on an advisory board for Clintara, a clinical research organization, and holds a patent from Repligen that yields no royalties. The other authors declare no conflict of interest.

\section{ACKNOWLEDGMENTS}

We thank Scott Schobel, MD and Steve Zlotnick, PharmD for their comments on the first draft of this manuscript.

\section{REFERENCES}

Adams W, Kendell RE, Hare EH, Munk-Jorgensen P (1993). Epidemiological evidence that maternal influenza contributes to the aetiology of schizophrenia. An analysis of Scottish, English, and Danish data. Br J Psychiatry 163: 522-534.

Akhondzadeh S, Tabatabaee M, Amini H, Ahmadi Abhari SA, Abbasi SH, Behnam B (2007). Celecoxib as adjunctive therapy in schizophrenia: a double-blind, randomized and placebocontrolled trial. Schizophr Res 90: 179-185.

Amital H, Barak V, Winkler RE, Rubinow A (2007). Impact of treatment with infliximab on serum cytokine profile of patients with rheumatoid and psoriatic arthritis. Ann NY Acad Sci 1110: 649-660.

Ashdown H, Dumont Y, Ng M, Poole S, Boksa P, Luheshi GN (2006). The role of cytokines in mediating effects of prenatal infection on the fetus: implications for schizophrenia. Mol Psychiatry 11: 47-55.

Behrens MM, Ali SS, Dao DN, Lucero J, Shekhtman G, Quick KL et al (2007). Ketamine-induced loss of phenotype of fast-spiking interneurons is mediated by NADPH-oxidase. Science 318: 1645-1647.

Behrens MM, Ali SS, Dugan LL (2008). Interleukin-6 mediates the increase in NADPH-oxidase in the ketamine model of schizophrenia. J Neurosci 28: 13957-13966.

Benveniste EN, Sparacio SM, Norris JG, Grenett HE, Fuller GM (1990). Induction and regulation of interleukin-6 gene expression in rat astrocytes. J Neuroimmunol 30: 201-212.

Bloomfield PS, Selvaraj S, Veronese M, Rizzo G, Bertoldo A, Owen DR et al (2016). Microglial activity in people at ultra high risk of psychosis and in schizophrenia: an [(11)C]PBR28 PET brain imaging study. Am J Psychiatry 173: 44-52.

Borrell J, Vela JM, Arevalo-Martin A, Molina-Holgado E, Guaza C (2002). Prenatal immune challenge disrupts sensorimotor gating in adult rats. Implications for the etiopathogenesis of schizophrenia. Neuropsychopharmacology 26: 204-215.

Brown AS, Begg MD, Gravenstein S, Schaefer CA, Wyatt RJ, Bresnahan M et al (2004). Serologic evidence of prenatal influenza in the etiology of schizophrenia. Arch Gen Psychiatry 61: 774-780.

Brown AS, Derkits EJ (2010). Prenatal infection and schizophrenia: a review of epidemiologic and translational studies. $A m \mathrm{~J}$ Psychiatry 167: 261-280.

Brown AS, Schaefer CA, Quesenberry CP Jr., Liu L, Babulas VP, Susser ES (2005). Maternal exposure to toxoplasmosis and risk of schizophrenia in adult offspring. Am J Psychiatry 162: 767-773.

Buka SL, Cannon TD, Torrey EF, Yolken RH (2008). Maternal exposure to herpes simplex virus and risk of psychosis among adult offspring. Biol Psychiatry 63: 809-815.

Buka SL, Tsuang MT, Torrey EF, Klebanoff MA, Bernstein D, Yolken RH (2001). Maternal infections and subsequent psychosis among offspring. Arch Gen Psychiatry 58: 1032-1037.

Burmester GR, Feist E, Kellner H, Braun J, Iking-Konert C, Rubbert-Roth A (2011). Effectiveness and safety of the interleukin 6-receptor antagonist tocilizumab after 4 and 24 weeks in patients with active rheumatoid arthritis: the first phase IIIb real-life study (TAMARA). Ann Rheum Dis 70: 755-759.

Collste K, Plaven-Sigray P, Fatouros-Bergman H, Victorsson P, Schain M, Forsberg A et al (2017). Lower levels of the glial cell marker TSPO in drug-naive first-episode psychosis patients as measured using PET and $\left[{ }^{11}\right.$ C]PBR28. Mol Psychiatry 22: $850-856$.

Fitzmaurice GM, Laird NM, Ware JH (2004). Applied Longitudinal Analysis. Wiley: Hoboken, NJ.

Fortier ME, Joober R, Luheshi GN, Boksa P (2004). Maternal exposure to bacterial endotoxin during pregnancy enhances amphetamine-induced locomotion and startle responses in adult rat offspring. J Psychiatr Res 38: 335-345. 
Frei K, Malipiero UV, Leist TP, Zinkernagel RM, Schwab ME, Fontana A (1989). On the cellular source and function of interleukin 6 produced in the central nervous system in viral diseases. Eur J Immunol 19: 689-694.

Ghodke Y, Joshi K, Chopra A, Patwardhan B (2005). HLA and disease. Eur J Epidemiol 20: 475-488.

Gilmore JH, Jarskog LF (1997). Exposure to infection and brain development: cytokines in the pathogenesis of schizophrenia. Schizophr Res 24: 365-367.

Green MF, Nuechterlein KH, Gold JM, Barch DM, Cohen J, Essock S et al (2004). Approaching a consensus cognitive battery for clinical trials in schizophrenia: the NIMH-MATRICS conference to select cognitive domains and test criteria. Biol Psychiatry 56: 301-307.

Guy W (1976). Clinical Global Impressions. In: Guy W(ed), ECDEU Assessment Manual for Psychopharmacology: Revised, ADM 76338. Department of Health, Education, and Welfare: Washington, DC. pp 217-222.

Hafizi S, Da Silva T, Gerritsen C, Kiang M, Bagby RM, Prce I et al (2017a). Imaging microglial activation in individuals at clinical high risk for psychosis: an in vivo PET study with $\left[{ }^{18} \mathrm{~F}\right] \mathrm{FEPPA}$. Neuropsychopharmacology 42: 2474-2481.

Hafizi S, Tseng HH, Rao N, Selvanathan T, Kenk M, Bazinet RP et al (2017b). Imaging microglial activation in untreated firstepisode psychosis: a PET study with $\left[{ }^{18} \mathrm{~F}\right] \mathrm{FEPPA}$. Am J Psychiatry 174: $118-124$.

Himmerich H, Schonherr J, Fulda S, Sheldrick AJ, Bauer K, Sack U (2011). Impact of antipsychotics on cytokine production in-vitro. J Psychiatr Res 45: 1358-1365.

Hopkins SJ, Rothwell NJ (1995). Cytokines and the nervous system. I: Expression and recognition. Trends Neurosci 18: 83-88.

Jones SA, Scheller J, Rose-John S (2011). Therapeutic strategies for the clinical blockade of IL-6/gp130 signaling. J Clin Invest 121: 3375-3383.

Kay SR, Sevy S (1990). Pyramidical model of schizophrenia. Schizophr Bull 16: 537-545.

Kemp AS, Schooler NR, Kalali AH, Alphs L, Anand R, Awad G et al (2010). What is causing the reduced drug-placebo difference in recent schizophrenia clinical trials and what can be done about it? Schizophr Bull 36: 504-509.

Kenk M, Selvanathan T, Rao N, Suridjan I, Rusjan P, Remington G et al (2015). Imaging neuroinflammation in gray and white matter in schizophrenia: an in-vivo PET study with $\left[{ }^{18} \mathrm{~F}\right]$-FEPPA. Schizophr Bull 41: 85-93.

Kinon BJ, Potts AJ, Watson SB (2011). Placebo response in clinical trials with schizophrenia patients. Curr Opin Psychiatry 24: 107-113.

Laan W, Grobbee DE, Selten JP, Heijnen CJ, Kahn RS, Burger H (2010). Adjuvant aspirin therapy reduces symptoms of schizophrenia spectrum disorders: results from a randomized, doubleblind, placebo-controlled trial. J Clin Psychiatry 71: 520-527.

Levine J, Schooler NR (1986). SAFTEE: a technique for the systematic assessment of side effects in clinical trials. Psychopharmacol Bull 22: 343-381.

Lewis DA, Hashimoto T, Volk DW (2005). Cortical inhibitory neurons and schizophrenia. Nat Rev Neurosci 6: 312-324.

Marder SR, Fenton W (2004). Measurement and treatment research to improve cognition in schizophrenia: NIMH MATRICS initiative to support the development of agents for improving cognition in schizophrenia. Schizophr Res 72: 5-9.

McBurney CA, Vina ER (2012). Racial and ethnic disparities in rheumatoid arthritis. Curr Rheumatol Rep 14: 463-471.

Miller BJ, Buckley P, Seabolt W, Mellor A, Kirkpatrick B (2011). Meta-analysis of cytokine alterations in schizophrenia: clinical status and antipsychotic effects. Biol Psychiatry 70: 663-671.

Miller BJ, Dias JK, Lemos HP, Buckley PF (2016). An open-label, pilot trial of adjunctive tocilizumab in schizophrenia. J Clin Psychiatry 77: 275-276.
Mortensen PB, Norgaard-Pedersen B, Waltoft BL, Sorensen TL, Hougaard D, Torrey EF et al (2007). Toxoplasma gondii as a risk factor for early-onset schizophrenia: analysis of filter paper blood samples obtained at birth. Biol Psychiatry 61: 688-693.

Muller N, Krause D, Dehning S, Musil R, Schennach-Wolff R, Obermeier $\mathrm{M}$ et al (2010). Celecoxib treatment in an early stage of schizophrenia: results of a randomized, double-blind, placebocontrolled trial of celecoxib augmentation of amisulpride treatment. Schizophr Res 121: 118-124.

Muller N, Riedel M, Scheppach C, Brandstatter B, Sokullu S, Krampe $\mathrm{K}$ et al (2002). Beneficial antipsychotic effects of celecoxib add-on therapy compared to risperidone alone in schizophrenia. Am J Psychiatry 159: 1029-1034.

Nitta M, Kishimoto T, Muller N, Weiser M, Davidson M, Kane JM et al (2013). Adjunctive use of nonsteroidal anti-inflammatory drugs for schizophrenia: a meta-analytic investigation of randomized controlled trials. Schizophr Bull 39: 1230-1241.

O'Callaghan E, Sham P, Takei N, Glover G, Murray RM (1991). Schizophrenia after prenatal exposure to 1957 A2 influenza epidemic. Lancet 337: 1248-1250.

Patel A, Zhu Y, Kuzhikandathil EV, Banks WA, Siegel A, Zalcman SS (2012). Soluble interleukin-6 receptor induces motor stereotypies and co-localizes with gp130 in regions linked to corticostriato-thalamo-cortical circuits. PLoS ONE 7: e41623.

Patterson PH (2009). Immune involvement in schizophrenia and autism: etiology, pathology and animal models. Behav Brain Res 204: 313-321.

Posner K, Brown GK, Stanley B, Brent DA, Yershova KV, Oquendo MA et al (2011). The Columbia-Suicide Severity Rating Scale: initial validity and internal consistency findings from three multisite studies with adolescents and adults. Am J Psychiatry 168: 1266-1277.

Raison CL, Rutherford RE, Woolwine BJ, Shuo C, Schettler P, Drake DF et al (2012). A randomized controlled trial of the tumor necrosis factor antagonist infliximab for treatment-resistant depression: the role of baseline inflammatory biomarkers. Arch Gen Psychiatry 1-11.

Revheim N, Medalia A (2004). The independent living scales as a measure of functional outcome for schizophrenia. Psychiatr Serv 55: 1052-1054.

Reyes TM, Coe CL (1998). The proinflammatory cytokine network: interactions in the CNS and blood of rhesus monkeys. Am J Physiol 274: R139-R144.

Rothwell NJ, Hopkins SJ (1995). Cytokines and the nervous system II: actions and mechanisms of action. Trends Neurosci 18: 130-136.

Rutherford BR, Pott E, Tandler JM, Wall MM, Roose SP, Lieberman JA (2014). Placebo response in antipsychotic clinical trials: a meta-analysis. JAMA Psychiatry 71: 1409-1421.

Samuelsson AM, Alexanderson C, Molne J, Haraldsson B, Hansell P, Holmang A (2006). Prenatal exposure to interleukin-6 results in hypertension and alterations in the renin-angiotensin system of the rat. J Physiol 575: 855-867.

Schwarz MJ (2003). Cytokines, neurophysiology, neuropsychology, and psychiatric symptoms. Dialog Clin Neurosci 5: 139-153.

Simpson GM, Angus JWS (1970). A rating scale for extrapyramidal side effects. Acta Psychiatr Scand 212(Suppl): 11-19.

Smith SE, Li J, Garbett K, Mirnics K, Patterson PH (2007). Maternal immune activation alters fetal brain development through interleukin-6. J Neurosci 27: 10695-10702.

Smolen JS, Aletaha D, Koeller M, Weisman MH, Emery P (2007). New therapies for treatment of rheumatoid arthritis. Lancet $\mathbf{3 7 0}$ : 1861-1874.

Wright HL, Bucknall RC, Moots RJ, Edwards SW (2012). Analysis of SF and plasma cytokines provides insights into the mechanisms of inflammatory arthritis and may predict response to therapy. Rheumatology (Oxford) 51: 451-459. 